\title{
Energy Finite Element Analysis Developments for Vibration Analysis of Composite Aircraft Structures
}

\author{
N. Vlahopoulos, and N. Schiller
}

\begin{abstract}
The Energy Finite Element Analysis (EFEA) has been utilized successfully for modeling complex structural-acoustic systems with isotropic structural material properties. In this paper, a formulation for modeling structures made out of composite materials is presented. An approach based on spectral finite element analysis is utilized first for developing the equivalent material properties for the composite material. These equivalent properties are employed in the EFEA governing differential equations for representing the composite materials and deriving the element level matrices. The power transmission characteristics at connections between members made out of non-isotropic composite material are considered for deriving suitable power transmission coefficients at junctions of interconnected members. These coefficients are utilized for computing the joint matrix that is needed to assemble the global system of EFEA equations. The global system of EFEA equations is solved numerically and the vibration levels within the entire system can be computed. The new EFEA formulation for modeling composite laminate structures is validated through comparison to test data collected from a representative composite aircraft fuselage that is made out of a composite outer shell and composite frames and stiffeners. NASA Langley constructed the composite cylinder and conducted the test measurements utilized in this work.
\end{abstract}

\section{INTRODUCTION}

The EFEA is an emerging technology for analyzing complex structural-acoustic systems [1-18]. The EFEA utilizes the spatially averaged vibrational or acoustic energy as a primary variable for developing governing differential equations for each wave type present within a system. The fundamental solution to the governing differential equations presents an exponential variation with space, which eliminates the requirement of using small finite elements at high frequencies. At discontinuities (i.e. connections between structural components, structural and acoustic interfaces, changes in material properties, changes in geometry, etc.) the primary variables in the EFEA become discontinuous. Joint matrices must be developed in order to introduce the power transfer characteristics at each discontinuity into the EFEA system of equations. For each finite element encountered at a discontinuity, the joint matrices represent relationships between the power flow at the element's boundaries and the corresponding EFEA primary variables. The joint matrices are developed from power transfer coefficients representative of the connections between the members encountered at the discontinuity. The derivation of power transfer coefficients is based on considering a connection between semiinfinite members, prescribing an impinging wave type originating from one of the members, and evaluating all transmitted and reflected wave types in all members. A library of element matrices and a library of joints can be combined for creating the EFEA model for a complex system and computing its structural-acoustic response.

This paper aims to further extend the capability of the EFEA method for computing the vibro-acoustic response of aircraft type of structures made out of composite laminates and/or composite sandwich panels. A Spectral Finite Element Method (SFEM) [19, 20] is used for evaluating the group speed $c_{g}^{*}$ and the damping $\eta^{*}$ due to the effectiveness of SFEM for taking into account the layer-wise composition of composite panels along with the shear deformation effects. The $c_{g}^{*}$ and $\eta^{*}$ values computed by the SFEM are used in the EFEA formulation for deriving the element level matrices of the composite materials. A method for deriving the power transmission coefficients between composite members is presented. The new method can take into account the shear deformation effects of composite plates. The power transfer coefficients are used for deriving the joint matrices in the EFEA formulation.

Theoretical aspects of these two formulations are presented in this paper. A numerical EFEA implementation for composite materials is utilized for analyzing the NASA Composite Test-bed Cylinder (CTC) and comparing simulation results to test data for the vibration at several bays of the cylinder. The CTC has longitudinal stiffeners (representing stringers) and circumferential stiffeners (representing frames) and represents the dynamic characteristics of a composite aircraft fuselage [22]. Excitation is applied through a shaker at different locations on the CTC and measurements are collected using a laser vibrometer at twelve bays of the cylinder. The results from the EFEA simulations are compared to test data in the frequency range that covers the $1 / 3$ octave bands of $800 \mathrm{~Hz}-$ $4,000 \mathrm{~Hz}$. The experimental set-up and the correlation study are discussed in this paper.

Page 1 of 10 


\section{DERIVATION OF GROUP SPEED AND DAMPING FOR COMPOSITES}

The $c_{g}^{*}$ and $\eta^{*}$ values computed by the SFEM are used in the EFEA formulation for composites for deriving the element level matrices. The basic steps of the SFEM approach are presented here. Figure depicts a plane wave propagating in the positive $x$ direction with frequency $\omega$ and wavenumber $k$ in a multilayer composite plate. The $\mathrm{x}$ direction comprises a reference for orienting the various layers. The through-thickness discretization in the $z$-axis along with the assumption of the harmonic wave motion in the $x$ direction results in the following form of displacement field, $\mathbf{u}^{T}=[u, v, w]$ at any point, $(x, y, z)$ within the plate,

$$
\mathbf{u}(x, z, t)=\mathbf{N}(z) \widehat{\mathbf{u}} e^{\mathrm{i}(\omega t-k x)}
$$

where $\mathbf{N}(z)$ is a matrix of shape functions and $\widehat{\mathbf{u}}$ is a vector of the nodal degrees of freedom of the form:

$$
\widehat{\mathbf{u}}=\left[\begin{array}{llll}
u_{1}, v_{1}, w_{1} & u_{2}, v_{2}, w_{2} & \cdots & u_{N_{e}+1}, v_{N_{e}+1}, w_{N_{e}+1}
\end{array}\right]^{T}
$$

where $N_{e}$ is the total number of linear finite elements through the thickness. It is noted that any of the displacement components in $\widehat{\mathbf{u}}$ may be complex numbers.

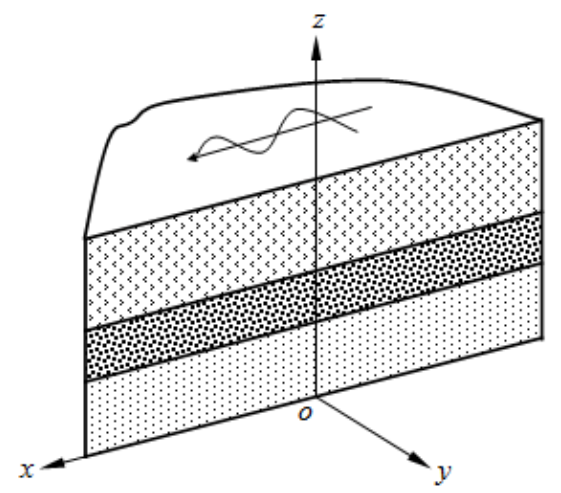

Figure 1: A plane wave propagation in a multilayer composite panel

The time-averaged total kinetic and potential energies, $\langle T\rangle$ and $\langle V\rangle$ are given by

$$
\langle T\rangle=\frac{\omega^{2}}{4} \int_{\Omega} \rho \mathbf{u}^{H} \mathbf{u} d \Omega ;\langle V\rangle=\frac{1}{4} \int_{\Omega} \boldsymbol{\sigma}^{H} \boldsymbol{\varepsilon} d \Omega
$$

where ${ }^{H}$ stands for the Hermitian transpose. Substitution of appropriate stress-strain and strain-displacement relations and the displacement field equation (1) into equation (3) results in:

$$
\langle T\rangle=\widehat{\mathbf{u}}^{H} \omega^{2} \mathbf{M} \widehat{\mathbf{u}} ;\langle V\rangle=\widehat{\mathbf{u}}^{H} \mathbf{K} \widehat{\mathbf{u}}
$$

where $\mathbf{K}$ and $\mathbf{M}$ are, respectively, the stiffness and mass matrices. The replacement of the spatial derivatives with respect to $x$ and $z$ by $-\mathrm{i} k$ and $d \mathbf{N} / d z$ can yield the expressions for the stiffness and mass matrices. Using Hamilton's principle results in:

$$
\left[\mathbf{K}(k)-\omega^{2} \mathbf{M}\right] \widehat{\mathbf{u}}=\mathbf{0}
$$

Since $\mathbf{K}=\mathbf{K}(k)$, for a prescribed circular frequency $\omega$, equation (5) can be solved for the eigenvalues $\omega^{2}$ and eigenvectors $\widehat{\mathbf{u}}$. The hysteretic damping model can be applied to each layer to yield the following form for the total time-averaged energy loss associated with an arbitrary wave type,

$$
\left\langle\underline{\pi}_{\text {diss }}\right\rangle=\sum_{l=1}^{N_{e}} \eta_{l} \omega\langle e\rangle_{l}
$$

where $\eta_{l}$ and $\langle e\rangle_{l}$ are, respectively, the structural loss factor and energy density of the $l$ th layer of multi-layered composites. Since $\langle e\rangle=\langle T\rangle+\langle V\rangle=2\langle V\rangle$, the damping loss factor associated with each propagating wave can be expressed as 


$$
\eta=\frac{\sum_{l=1}^{N_{e}} \eta_{l} \widehat{\mathbf{u}}^{H} \mathbf{K}^{(l)} \widehat{\mathbf{u}}}{\widehat{\mathbf{u}}^{H} \mathbf{K} \widehat{\mathbf{u}}}
$$

Here, the distinction should be made that $\mathbf{K}^{(l)}$ is the stiffness matrix of the $l$ th layer and $\mathbf{K}$ is the assembled global stiffness matrix of all layers. Equation (7) is derived for each wave heading angle $\theta_{i}$, and thus the angle-average must be considered for accounting for the full range of wave propagation directions when deriving the angle averaged damping loss factor as follows:

$$
\eta^{*}=\frac{\int_{\Theta} \eta\left(\theta_{i}\right) d \theta_{i}}{\int_{\Theta} d \theta_{i}}
$$

The variation of the angle $\theta_{i}$ is obtained by rotating the relative orientation of the x-axis with respect to the composite material (Figure 1). The circular frequency for the wave number $k$ can be written in terms of the Rayleigh quotient as

$$
\omega^{2}=\frac{\widehat{\mathbf{u}}^{H} \mathbf{K}(k) \widehat{\mathbf{u}}}{\widehat{\mathbf{u}}^{H} \mathbf{M} \widehat{\mathbf{u}}}
$$

Figure 2 shows the wavenumber of an arbitrary wave propagating in an anisotropic medium as a function of wave heading, $\theta_{i}$. The distribution and the direction of energy flow in anisotropic media like composites exhibit directional dependence, thus the direction of the energy flow, depicted as $\theta_{e}$ in Figure 2, is usually different from the wave heading, $\theta_{i}$. Thus, an appropriate correction must be introduced in the calculation of the group speed.

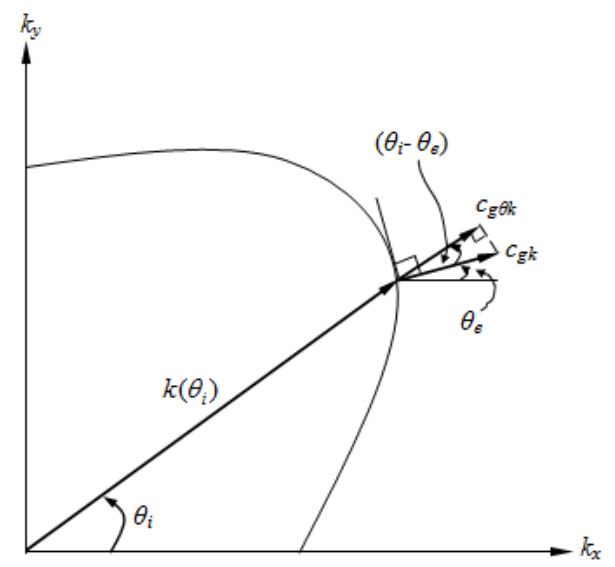

Figure 2: Wavenumber as a function of wave heading in the $k$ (wave number) plane

The group speed in the direction of wave propagation is $c_{g \theta}=d \omega / d k$, and the differentiation of equation (9) with respect to the wavenumber, $k$, results in:

$$
c_{g \theta}=\frac{\widehat{\mathbf{u}}^{H}(\partial \mathbf{K}(k) / \partial k) \widehat{\mathbf{u}}}{2 \omega \widehat{\mathbf{u}}^{H} \mathbf{M} \widehat{\mathbf{u}}}
$$

Referring to Figure 2, the following relationship holds:

$$
c_{g}=\frac{c_{g \theta}}{\cos \left(\theta_{i}-\theta_{e}\right)}
$$

where the heading of group speed, $\theta_{e}$ may be derived from the geometric interpretation of the wavenumber curve shown in Figure 2

$$
\tan \theta_{e}=-\frac{\partial k_{x} / \partial \theta_{i}}{\partial k_{y} / \partial \theta_{i}}=-\frac{\left(\partial k\left(\theta_{i}\right) / \partial \theta_{i}\right) \cos \theta_{i}-k\left(\theta_{i}\right) \sin \theta_{i}}{\left(\partial k\left(\theta_{i}\right) / \partial \theta_{i}\right) \sin \theta_{i}+k\left(\theta_{i}\right) \cos \theta_{i}}
$$

Therefore, the angle-averaged group speed can be evaluated for the full range of wave propagation directions as follows: 


$$
c_{g}{ }^{*}=\frac{\int_{\Theta} c_{g}\left(\theta_{i}\right) d \theta_{i}}{\int_{\Theta} d \theta_{i}}
$$

In the EFEA formulation for composites, $c_{g}^{*}$ and $\eta^{*}$ from Equations (13) and (8) respectively, are used when deriving the element level EFEA matrices.

\section{POWER TRANSFER COEFFICIENTS BETWEEN COMPOSITE MEMBERS}

The derivation of power transfer coefficients is based on considering a connection between semi-infinite members, prescribing an impinging wave type originating from one of the members, and evaluating all transmitted or reflected wave types in all members. Once available, the power transfer coefficients are used for computing the EFEA joint matrices which connect elements across a discontinuity in the EFEA system of equations. The formulation for computing power transfer coefficients between composite members is presented here. It must be noted, that this formulation accounts for First order Shear Deformation Theory (FSDT) effects, which are important when considering sandwich composite panels. The extended wave dynamic stiffness matrix approach [21] is used to calculate the wave power transfer coefficients of coupled composite members.

Figure 3 presents a junction consisting of $\mathrm{N}$ semi-infinite composite members. The coordinate systems and the translational and rotational displacements are also presented in Figure 3.

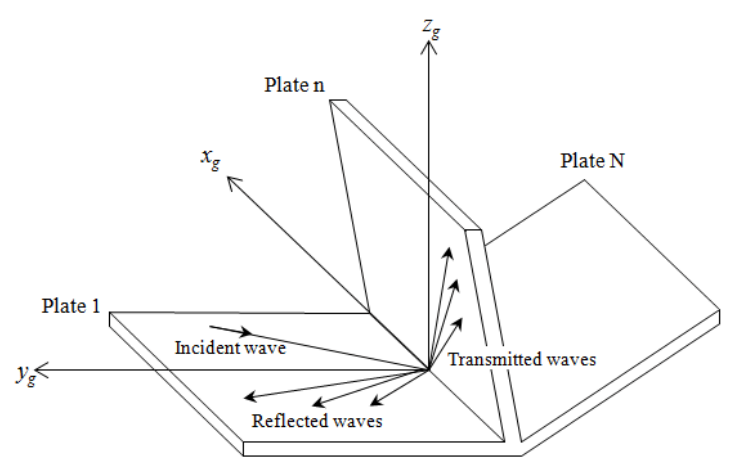

(a)

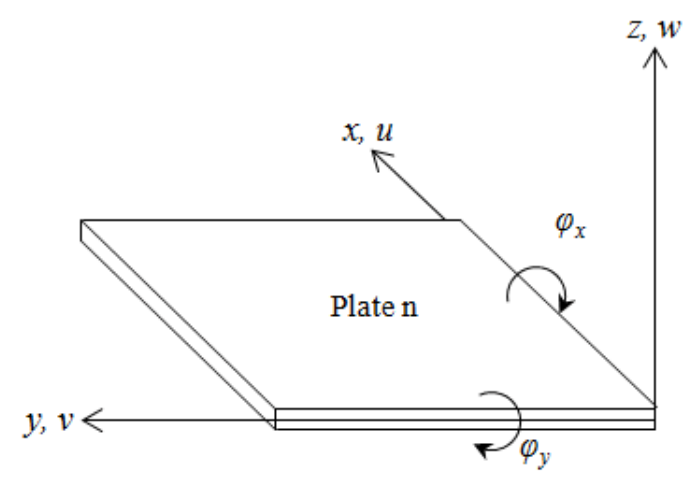

(b)

Figure 3: (a) A general N-plate junction and global coordinate system; (b) local coordinate system and displacements for plate $\mathrm{n}$

For either a laminated or a sandwich composite member, the FSDT allows writing the dynamic equations of motion in the following matrix-vector form: 


$$
\mathbf{L u}=\mathbf{0}
$$

where $\mathbf{u}=\left[u, v, w, \varphi_{x}, \varphi_{y}\right]^{T}$ and $\mathbf{L}$ is a linear differential operator which can be expressed in terms of elastic constants $A_{i j}, B_{i j}$ and $D_{i j}$ and inertial properties $I_{\alpha}(\alpha=0,1,2)$. Substitution of a wave form, $\mathbf{u}=\widehat{\mathbf{u}} e^{-i k_{x} x+\lambda y+i \omega t}$ into equation (14) yields

$$
\left(\lambda^{2} \mathbf{b}_{2}+\lambda \mathbf{b}_{1}+\mathbf{b}_{0}\right) \widehat{\mathbf{u}}=0
$$

where $\mathbf{b}_{0}, \mathbf{b}_{1}$, and $\mathbf{b}_{2}$ can be obtained from $\mathbf{L}$ by replacing $\frac{\partial}{\partial x}, \frac{\partial}{\partial y}$ and $\frac{\partial}{\partial t}$ by $\left(-i k_{x}\right), \lambda$, and $\omega$. This quadratic eigenvalue problem can be solved for five pairs of $\lambda$ 's and $\widehat{\mathbf{u}}$ 's for given $k_{x}$ and $\omega$. Then, the edge displacements, $\mathbf{d}_{\mathrm{y}}$ and the corresponding elastic tractions, $\mathbf{t}_{\mathrm{y}}$ of each plate can be expressed in terms of the reflected/transmitted wave amplitudes $\mathbf{c}=\left[c_{1}, c_{2}, c_{3}, c_{4}, c_{5}\right]^{\mathbf{T}}$ and an incident wave amplitude $c_{0}$ as follows

$$
\begin{aligned}
\mathbf{d}_{\mathrm{y}} & =\left[\begin{array}{llll}
\mathbf{d}_{\mathrm{y} 1} & \mathbf{d}_{\mathrm{y} 2} & \cdots & \mathbf{d}_{\mathrm{y} 5}
\end{array}\right] \mathbf{c}+\mathbf{d}_{\mathrm{y} 0} c_{0} \\
\mathbf{t}_{\mathrm{y}} & =\left[\begin{array}{llll}
\mathbf{t}_{\mathrm{y} 1} & \mathbf{t}_{\mathrm{y} 2} & \cdots & \mathbf{t}_{\mathrm{y} 5}
\end{array}\right] \mathbf{c}+\mathbf{t}_{\mathrm{y} 0} c_{0}
\end{aligned}
$$

where $\mathbf{d}_{\mathrm{y} i}$ and $\mathbf{t}_{\mathrm{y} i}(i=1,2, \ldots, 5)$ represent edge displacements and elastic tractions due to the $i$ th transmitted or reflected wave and $\mathbf{d}_{\mathrm{y} 0}$ and $\mathbf{t}_{\mathrm{y} 0}$ are those for incident waves. Equations (16) and (17) can be combined to give

$$
\mathbf{t}_{\mathrm{y}}=\mathbf{K} \mathbf{d}_{\mathrm{y}}-\mathbf{f}_{\mathrm{y} 0}
$$

where $\mathbf{K}=\left[\begin{array}{llll}\mathbf{d}_{\mathrm{y} 1} & \mathbf{d}_{\mathrm{y} 2} & \cdots & \mathbf{d}_{\mathrm{y} 5}\end{array}\right]^{-1}\left[\begin{array}{lllll}\mathbf{t}_{\mathrm{y} 1} & \mathbf{t}_{\mathrm{y} 2} & \cdots & \mathbf{t}_{\mathrm{y} 5}\end{array}\right]$ is the wave dynamic stiffness matrix and $\mathbf{f}_{\mathrm{y} 0}=\mathbf{K} \mathbf{d}_{\mathrm{y} 0}-\mathbf{t}_{\mathrm{y} 0}$ is the force due to the incident wave. It should be noted that $\mathbf{f}_{\mathrm{y} 0}=\mathbf{0}$ in the plates where no incident wave exists. By implying subscript " $y$ ” for the remaining discussion, equation (18) can be rewritten for the $n$th plate as

$$
\mathbf{t}_{\mathrm{n}}=\mathbf{K}_{\mathrm{n}} \mathbf{d}_{\mathrm{n}}-\mathbf{f}_{\mathrm{n}}
$$

The wave dynamic matrix is assembled to yield an equation for the computation of power transmission coefficients. Since $\mathbf{d}_{\mathrm{n}}$ and $\mathbf{t}_{\mathrm{n}}$ are defined in the local coordinate system, they need to be transformed to those at the common junction defined in the global coordinate system, $\mathbf{d}_{J}$ and $\mathbf{t}_{J}$ as follows:

$$
\mathbf{d}_{\mathrm{n}}=\mathbf{R}_{\mathrm{n}} \mathbf{d}_{J} ; \mathbf{t}_{J}=\mathbf{R}_{\mathrm{n}}^{\mathrm{T}} \mathbf{t}_{\mathrm{n}}
$$

where $\mathbf{R}_{\mathrm{n}}$ is a simple coordinate transformation matrix consisting of $\cos \psi_{n}$ and $\sin \psi_{n}$. Since the force equilibrium equation for the $N$ plates connected through a structural joint is $\sum_{\mathrm{n}=1}^{\mathrm{N}} \mathbf{R}_{n}^{\mathrm{T}} \mathbf{t}_{n}=\mathbf{0}$, using equations (19) and (20) results in:

$$
\left[\sum_{\mathrm{n}=1}^{\mathrm{N}} \mathbf{R}_{\mathrm{n}}^{\mathrm{T}} \mathbf{K}_{\mathrm{n}} \mathbf{R}_{\mathrm{n}}\right] \mathbf{d}_{J}=\left[\sum_{\mathrm{n}=1}^{\mathrm{N}} \mathbf{R}_{\mathrm{n}}^{\mathrm{T}} \mathbf{f}_{\mathrm{n}}\right]
$$

where $\mathbf{d}_{J}$ can be evaluated from Equation (21). Using equation (16) the wave amplitudes are evaluated:

$$
\mathbf{c}=\left[\begin{array}{llll}
\mathbf{d}_{\mathrm{y} 1} & \mathbf{d}_{\mathrm{y} 2} & \cdots & \mathbf{d}_{\mathrm{y} 5}
\end{array}\right]^{-1}\left(\mathbf{d}_{\mathrm{y}}-\mathbf{d}_{\mathrm{y} 0} c_{0}\right)
$$

Then, the power transfer coefficients can be readily calculated from:

$$
\tau_{i j}(\omega, \theta)=\frac{\left|c_{j}\right|^{2}}{\left|c_{0 i}\right|^{2}}
$$

where $c_{j}$ is the amplitude of a reflected or transmitted wave type $j$ for the given $c_{0 i}$, which is the amplitude of an incident wave type $i$ with frequency $\omega$ and an incidence angle of $\theta$.

The above computed transmission coefficients are highly dependent on the angle of incidence, $\theta$ for anisotropic media (i.e. composite panels). Thus, the power transfer coefficients are obtained through averaging of the power transmission coefficients with respect to the angle of incidence: 


$$
\left\langle\tau_{i j}\right\rangle=\int_{\Theta} \frac{\tau_{i j}(\theta) c_{g y}(\theta)}{c(\theta) c_{g \theta}(\theta)} d \theta / \int_{\Theta} \frac{c_{g y}(\theta)}{c(\theta) c_{g \theta}(\theta)} d \theta
$$

The power transfer coefficients are used for creating the EFEA joint matrices which connect elements across a discontinuity. Composite material properties can be considered for the components connected across a discontinuity based on the formulation presented in this Section.

\section{SIMULATIONS AND COMPARISON TO TEST DATA}

The EFEA capability for modeling the response of composite aircraft type structures in high frequencies due to structure-borne excitation is validated by comparing simulation results to test data collected using the NASA CTC. Test results are compared to EFEA simulations in the frequency range that covers $1 / 3$ octave bands from $800 \mathrm{~Hz}-4,000 \mathrm{~Hz}$. The description of the test set-up, and the correlation between the EFEA and the test data are discussed in this Section.

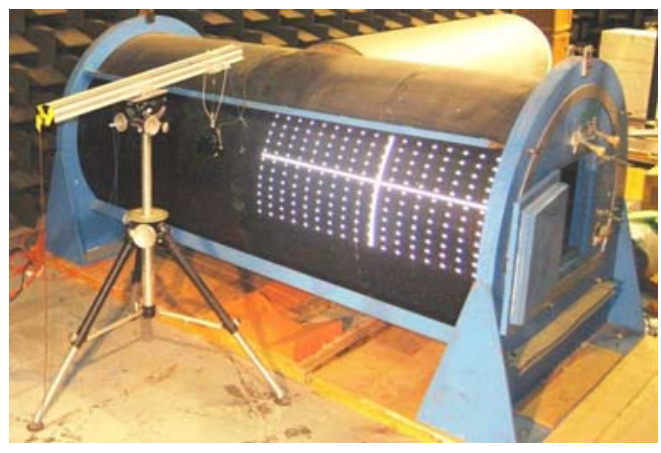

(a)

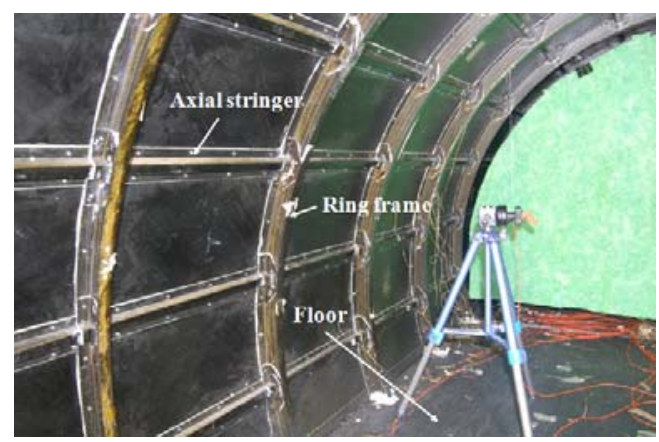

(b)

Figure 4: Exterior (a) and interior (b) of the NASA composite test-bed cylinder

The NASA CTC structure, presented in Figure 4, is a floor-equipped stiffened composite cylinder that is $1.676 \mathrm{~m}$ in diameter and $3.658 \mathrm{~m}$ long. The $1.7 \mathrm{~mm}$ thick shell is made of carbon fiber filaments embedded in an epoxy resin. Specifically, the shell is made of 9 layers of $0.189 \mathrm{~mm}$ thick T500-tape with a ply sequence of $\pm 45, \pm 32,90, \mp 32$, $\mp 45$. The shell is reinforced by orthogonal supports, referred to as ring frames and stringers that are riveted and bonded to the skin. Ten J-section ring frames and twenty-two hat section stringers segment the cylinder into 198 rectangular bays with nominal dimensions of $0.203 \mathrm{~m}$ by $0.381 \mathrm{~m}$. The entire structure is supported by two $88.9 \mathrm{~mm}$ thick particle board end caps. Details regarding the construction of the cylinder can be found in [22].

The structure was excited by a single electromagnetic shaker suspended from an adjustable excitation stand. The shaker was attached at ten different locations as indicated with red dots in Figure 5. Two sets of four excitation locations placed on the shell comprise groups A and B while excitation locations C and D are on axial stiffeners. The multiple excitation locations within groups A and B enabled spatial averaging to smooth out local variations in the driven bay. Multiple experiments were also conducted with the shaker attached at the same point to evaluate response sensitivity to unintentional variations in shaker alignment and preload. An impedance head was placed between the stinger and the structure to measure the axial acceleration and force at the drive point. The normal shell Page 6 of 10 
velocity was measured using a laser vibrometer. The scanning laser vibrometer measured sidewall velocity at 204 points within the measurement area (shaded area) shown in Figure 5.

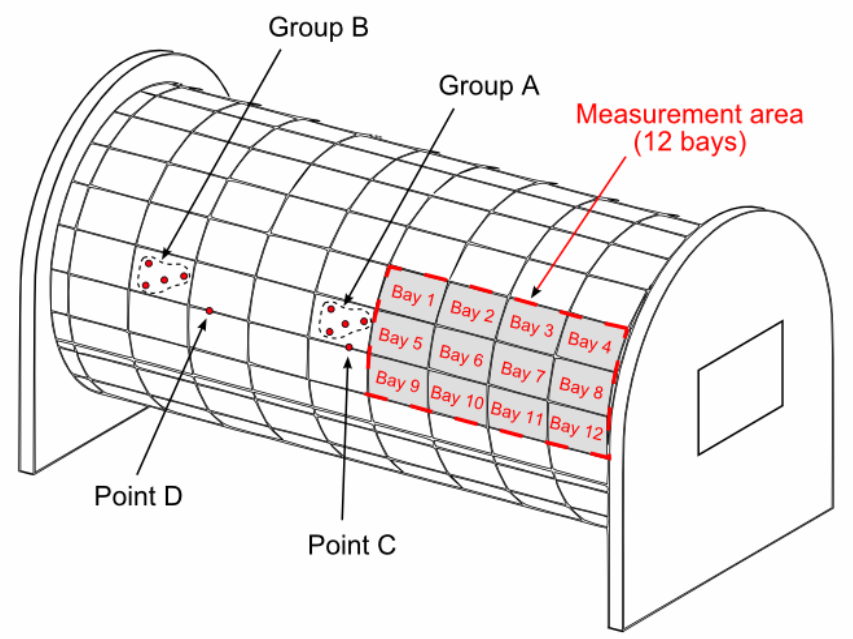

Figure 5: Drive point locations and measurement area (shaded area)

The measured vibro-acoustic response of the test structure is compared to numerical results obtained using the EFEA formulation presented in this paper. The EFEA model of the NASA CTC is shown in Figure 6. The comparison between measurements and predictions is performed on the twelve shaded bays shown in Figure 5. Specifically, the time and space averaged energy density in each of the twelve bays is calculated from measurements and compared to the EFEA results. In order to facilitate the comparison, both the predictions and the measurements are normalized with respect to the input power, $\pi_{i n}$. The final comparison is performed using the averaged normalized energy density, calculated as

$$
d B=10 \log \left(\frac{\sum_{j=1}^{N_{c}}\left(\frac{\rho\langle\underline{v}\rangle^{2}}{\left\langle\underline{\pi}_{i n}\right\rangle}\right)}{N_{c}}\right)
$$

where $N_{c}$ is the number of test cases (defined as the product between the number of repeated experiments and the number of excitation locations). $\langle\underline{v}\rangle$ and $\left\langle\underline{\pi}_{i n}\right\rangle$ are the averaged normal shell velocity and the averaged input power. It should be noted that the energy density is related to the velocity squared as $\langle\underline{e}\rangle=\rho\langle\underline{v}\rangle^{2}$.

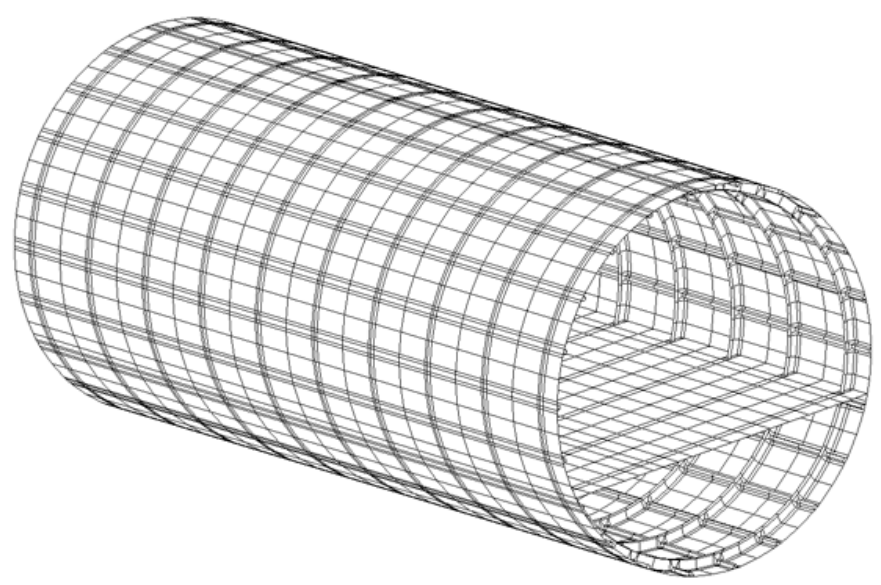

Figure 6. EFEA model for NASA CTC (without the end caps included in the figure) 
Figure 7 shows the difference (in $\mathrm{dB}$ ) between measurements and predictions in the $2000 \mathrm{~Hz}$ 1/3-octave band. The twelve rectangles in each subplot correspond to the twelve measurement bays depicted in Figure 5. The four subplots correspond to the different excitation groups. In this case, positive values indicate that the measurements are higher than the predictions. In most cases, good correlation exists between the measured data and predictions with maximum discrepancies of $6 \mathrm{~dB}$ in select bays. Although not shown, similar results were observed in the other 1/3-octave bands from 800 through 4,000 Hz.

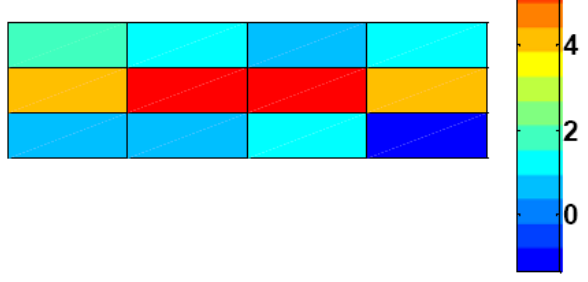

$\mathrm{dB}$ difference between EFEA and test results (Drive Point Group A)

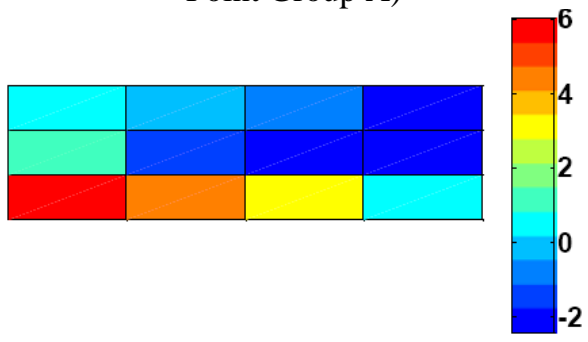

$\mathrm{dB}$ difference between EFEA and test results (Drive Point C)

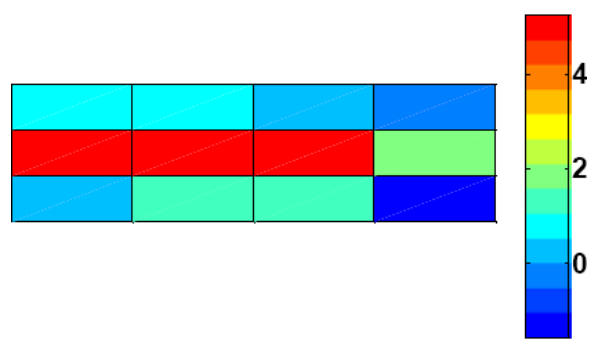

dB difference between EFEA and test results (Drive Point Group B)

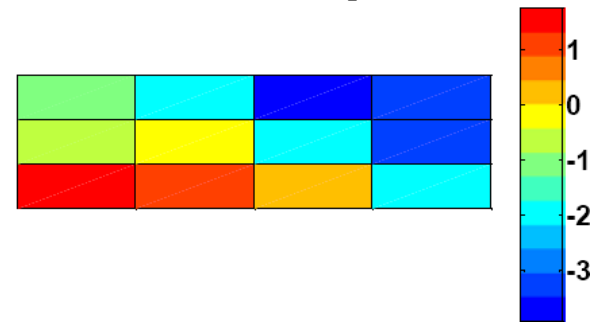

dB difference between EFEA and test results (Drive Point D)

Figure 7: Difference (dB) in normalized energy density in the $2000 \mathrm{~Hz} 1 / 3$ octave band

Figure 8 presents the differences in the energy density between test and simulations for each panel, averaged over the entire frequency range between the $800 \mathrm{~Hz}$ and $4,000 \mathrm{~Hz} 1 / 3$ octave bands for the four excitation locations. The frequency averaged difference between EFEA and test is computed as:

$$
\frac{\sum_{j=1}^{N_{f}}\left|d B_{E F E A}-d B_{t e s t}\right|}{N_{f}}
$$

where, $N_{f}$ is the number of center frequencies within the range of $1 / 3$ octave bands from $800-4000 \mathrm{~Hz}$. The calculation of $d B_{E F E A}$ and $d B_{\text {test }}$ is performed according to Equation (25). The majority of the differences are below $3 \mathrm{~dB}$, which comprises a very good correlation. Further, in the panels where isolated $5 \mathrm{~dB}$ differences are observed, the adjacent panels exhibit much better agreement, which indicates a lack of any physics based modeling errors. 


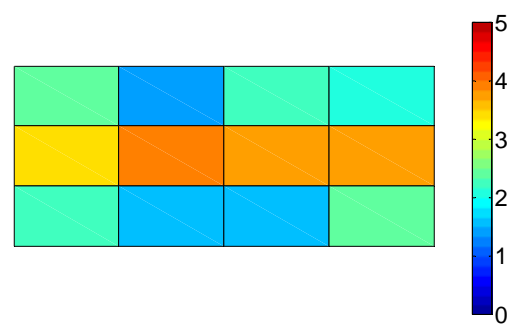

$\mathrm{dB}$ difference between EFEA and test results (Drive Point Group A)

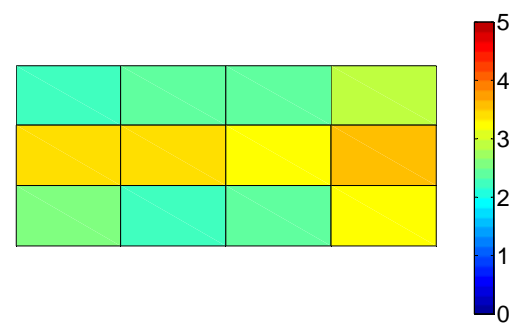

$\mathrm{dB}$ difference between EFEA and test results (Drive Point C)

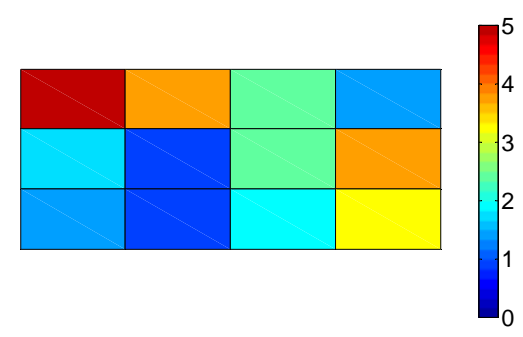

dB difference between EFEA and test results (Drive Point Group B)

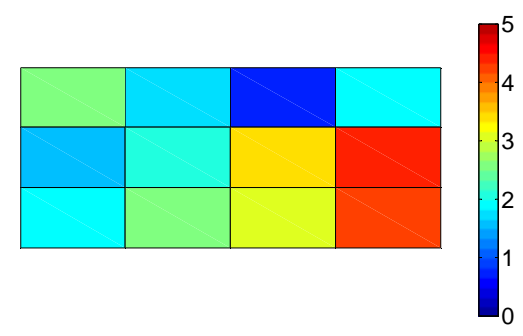

$\mathrm{dB}$ difference between EFEA and test results (Drive Point D)

Figure 8: Difference of normalized energy densities over measurement bays (in dB)

\section{SUMMARY}

A comprehensive formulation is presented in this paper for evaluating material properties and power transfer coefficients that can be used in an EFEA formulation for modeling composite structural systems. The new developments are employed for modeling a composite stiffened cylinder representative of a composite aircraft fuselage. Simulation results are compared successfully to test data, demonstrating how the new EFEA developments can be used for modeling a complex structural system made out of composite materials.

\section{REFERENCES}

1. J.E. Huff Jr. and R.J. Bernhard, "Prediction of High Frequency Vibrations in Coupled Plates Using Energy Finite Elements," Proceedings, Inter-Noise 95, 1995, Newport Beach, CA,USA, 1221-1126.

2. R. J. Bernhard and J. E. Huff, "Structural-Acoustic Design at High Frequency Using the Energy Finite Element Method,” Journal of Vibration and Acoustics, 121(7) (1999) 295-301.

3. S. Wang, and R. J. Bernhard, "Energy Finite Element Method (EFEM) and Statistical Energy Analysis (SEA) of a Heavy Equipment Cab,” SAE Paper 1999-01-1705.

4. K. K. Choi, and J. Dong, "Parametric Design Sensitivity Analysis of High Frequency Structural-Acoustic Problems Using Energy Finite Element Method,” ASME 2003 Design Engineering Technical Conferences and Computers and Information in Engineering Conference, Chicago, Illinois, USA, 2003, DETC2003/DAC-48753.

5. N.H. Kim, J. Dong, K. K. Choi, "Energy Flow Analysis and design Sensitivity of Structural Problems at High-Frequencies," Journal of Sound and Vibration, vol. 269, 2004, pp. 213-250.

6. Weiguo Zhang and S. T. Raveendra. High Frequency Vibro-Acoustic Analysis Using Energy Finite Element Method, SAE 2009 world congress, Detroit, Michigan, USA, SAE International, 2009-01-0771.

7. W. Zhang and S. T. Raveendra. Sound Package Analysis using Energy Finite Element Method, Noise and Vibration Conference and Exhibition, St. Charles, Illinois, USA, SAE International, 2007-01-2309. 
8. E.R.O. Santos, V.S. Pereira, J.R.F. Arruda and J.M.C. Dos Santos, "Structural Damage Detection using Energy Flow Models,” Shock and Vibration, 15 (2008) 217-230.

9. D.H. Park, S.Y. Hong, H.G. Kil, J.J. Jeon, Power flow models and analysis of in-plane waves in finite coupled thin plates, Journal of Sound and Vibration 244 (4) (2001) 651-668.

10. S. Cho, C-Y Park, Y-H Park, S-Y Hong, “Topology Design Optimization of Structures at High Frequencies Using Power Flow Analysis,” Journal of Sound and vibration, Vol. 298, Nov. 2006, pp. 206-220.

11. W. Zhang, N. Vlahopoulos, K. Wu, A. Wang "High Frequency Vibration Analysis of Stiffened Plates under Heavy Fluid Loading by an Energy Finite Element Analysis Formulation,” Finite Elements in Analysis and Design, v 41, n 11-12, June, 2005, p 10561078.

12. W. Zhang, N. Vlahopoulos, K. Wu, “An Energy Finite Element Formulation for High Frequency Vibration Analysis of Externally Fluid-Loaded Cylindrical Shells with Periodic Circumferential Stiffeners subjected to Axi-Symmetric Excitation” Journal of Sound and Vibration, Vol. 282, April 22 2005, p. 679-700.

13. A. Wang, N. Vlahopoulos, R. D. Buehrle, and J. Klos, "Energy Finite Element Analysis of the NASA Aluminum Test-Bed Cylinder,” SAE Paper No. 2005-01-2372, 2005 SAE Noise and Vibration Conference, Traverse City, MI.

14. G. Zhang, and N. Vlahopoulos, "Validation of an EFEA formulation for computing the vibrational response of complex structures,” SAE Paper No. 2007-01-2324, SAE 2007 Transactions Journal of Passenger Cars - Mechanical Systems.

15. N. Vlahopoulos, K. Wu, "Structural-Acoustic Simulations of Naval Vehicles using an Energy Finite Element Method,” MAST Americas, 2010, June 2010, Crystal City, VA.

16. Comet Technology Corporation, “Comet EnFlow User’s Manual,” 2007.

17. Michigan Engineering Services LLC, “MES/EFEA 2.0: User’s Manual,” 2007.

18. MSC Software Corporation, “MD Nastran 2010, EFEA User Guide,” 2010.

19. G. R. Liu, Z. C. Xi, 2002 Elastic Waves in Anisotropic Laminates. CRC Press

20. P. J. Shorter, Wave propagation and damping in linear viscoelastic laminates, Journal of Acoustical Society of America, 115 (2004) 1917-1925.

21. I. Bosmans, P. Mees, G. Vermeir, "Structure-borne sound transmission between thin orthotropic plates: Analytical Solutions," Journal of Sound and vibration, Vol. 191, 1996, pp. 75-90.

22. A. C. Jackson, F. J. Balena, W. L. LaBarge, G. Pei, W. A. Pitman, and G. Wittlin, Transport composite fuselage technology: Impact dynamics and acoustic transmission, NASA Contractor Report 4035, 1986.

\section{ACKNOWLEDGMENTS}

This work was supported by NASA grant NNX07AD06A. 